\title{
A Comparative Study for Telerobotic Surgery Using Free Hand Gestures
}

\author{
Tian Zhou, Maria E. Cabrera, Juan P. Wachs \\ Purdue University \\ Thomas Low \\ SRI International \\ and \\ Chandru Sundaram \\ Indiana University
}

\begin{abstract}
This research presents an exploratory study among touch-based and touchless interfaces selected to teleoperate a highly dexterous surgical robot. The possibility of incorporating touchless interfaces into the surgical arena may provide surgeons with the ability to engage in telerobotic surgery similarly as if they were operating with their bare hands. On the other hand, precision and sensibility may be lost. To explore the advantages and drawbacks of these modalities, five interfaces were selected to send navigational commands to the Taurus robot in the system: Omega, Hydra, and a keyboard. The first represented touch-based, while Leap Motion and Kinect were selected as touchless interfaces. Three experimental designs were selected to test the system, based on standardized surgically related tasks and clinically relevant performance metrics measured to evaluate the user's performance, learning rates, control stability, and interaction naturalness. The current work provides a benchmark and validation framework for the comparison of these two groups of interfaces and discusses their potential for current and future adoption in the surgical setting.
\end{abstract}

Keywords: human-robot interaction, teleoperation, touchless gestures, robot-assisted surgery, sensory substitution

\section{Introduction}

Medical advances in science and technology in recent years have made possible the inclusion of novel devices, interventions, and interfaces to interact with information, in order to enhance clinical outcomes while maximizing the patients' (and clinical workers') experience. One example is the progressive incursion of robotic platforms into surgery, whether as an assistive device or as a slave-console of a more complex teleoperation system (Greer, Newhook, \& Sutherland, 2008; $\mathrm{Hu}$ et al., 2011). Expressiveness and naturalness of hand motions present when performing direct surgery (O’Hara, Harper, Mentis, Sellen, \& Taylor, 2013) is not found in current telerobotic surgical systems. Instead of using bare hands to operate, teleoperated surgical robots rely on a set of controls, acting as interfaces between the surgeons and robots, which translate the surgeon's

Authors retain copyright and grant the Journal of Human-Robot Interaction right of first publication with the work simultaneously licensed under a Creative Commons Attribution License that allows others to share the work with an acknowledgement of the work's authorship and initial publication in this journal.

Journal of Human-Robot Interaction, Vol. 5, No. 2, 2016, Pages 1-27. DOI 10.5898/JHRI.5.2.Zhou 
movements to robotic commands. These consoles have a rather large footprint and are not as close to the patient as surgeons are in direct surgery. Additionally, surgeons are uncoupled from the rest of the surgical team (Frankel, Leonard, \& Denham, 2006).

Several aspects related to the use of the body and hands as surgical tools during surgery in the operating room (OR) are fundamentally different in common teleoperating systems. In such settings, the surgeon's hands are usually used as navigation terminals leading to use their movements as a means of control; such movements are very different from what surgeons normally use when they are in front of the patient (Mitra \& Acharya, 2007). The problem is that hands are not only vertices representing trajectories in a three dimensional space, but parts of the body capable of conveying intent, expression, and skillful action while gathering tactile information (Nehaniv et al., 2005).

In interpersonal communication, speech and body language are used to express emotion, mood, attitude, and attention (Jaimes \& Sebe, 2005), as much as it happens when humans interact with objects and their environment. This resonates in studies within the field of embodied cognition (Lakoff \& Johnson, 2003) in psychology, which postulates that cognition is shaped by aspects of the body. Embodied interaction is also related to the way people interact mentally and physically with their surroundings. All these are key components of communication and thus are aligned with performing in-situ surgery. For example, if the intent expressed through gestures is lost, there is a lack of communication grounding among the surgical team that could potentially lead to surgical complications and unnecessary delays (Lingard, Reznick, Espin, Regehr, \& DeVito, 2002; Prentice, 2007). While telesurgical systems, such as da Vinci, allow more than one surgeon to operate on the patient simultaneously, there is no mechanism to facilitate nonverbal communication between them, as in direct surgery. In such a context, the proxemics aspect of teamwork in teleoperated robotic surgery is lost (Moore, Butt, Ellis-Clarke, \& Cartmill, 2010; Whittaker, 2003). Improving communications in the operating room, especially when new technology is involved (Webster \& Cao, 2006), may lead to a better experience for the patient, which could involve fewer complications and faster overall recovery periods. These are all good reasons to explore the potential and feasibility of gestural interfaces. The ultimate interface would be that one that allows surgeons to use free hand gestures to operate the robotic platforms, similar to the way that they would use their hands during in-situ surgery. This could also offer advantages, such as maintaining asepsis (Hartmann et al., 2004; Schultz, Gill, Zubairi, Huber, \& Gordin, 2003). Touch-based interfaces were found to be correlated to incidence of infections in the operating room. Mapping embodied interaction to specific robotic commands could result in tactile perception, communication grounding in the surgical setting, and rich expression (Visell, 2009). Recent progress in gaming technologies provides innovative opportunities for motion tracking and human-machine interaction. In the field of healthcare, sensors like Microsoft $₫$ Kinect (2015) have been used for detecting postures in rehabilitation (Huang, 2011), gesture recognition for image browsing (Jacob, Cange, Packer, \& Wachs, 2012; Jacob, Wachs, \& Packer, 2013; Wachs et al., 2008), and as means for surgical robot control (Kim, Kim, Selle, Shademan, \& Krieger, 2014; Kim, Leonard, Shademan, Krieger, \& Kim, 2014; Zhou, Cabrera, \& Wachs, 2014, 2015).

However, a direct consequence of performing free hand gestures is lack of force-feedback provided by contact with a surface, thus leading to a lack of precision, sensitivity, and context. Sensory substitution paradigm provides the ground to partially mitigate this issue, leveraging sound and visual cues to convey information about the task being performed. Sensory substitution has been successfully used for many years helping people who are fully or partially deficient in one or more of their sensory systems (Mann, 1974; Rovira, Gapenne, \& Ammar, 2010). Further work also shows that sensory substitution, in the form of visual feedback, auditory feedback, or both, confers quantifiable advantages in applied force accuracy and consistency compared to no feedback at all during the performance of a simple surgical task (Kitagawa, Dokko, Okamura, \& Yuh, 2005). 
This work presents a study among different interfaces used to teleoperate a highly dexterous robot to complete surgically related tasks. The selected interfaces are comprised by both touchbased and touchless, relying on commodity sensors, traditional interfaces, and gaming technologies.

Fig. 1 shows the overall implementation of our system. All selected tasks have been recognized as part of either the Fundamentals in Laparoscopic Surgery (FLS) (Dunkin \& Wilcox, 2014) or selected by the University of Texas Southwestern (UTSW), among nine specific experiments, increasing in complexity and required level of dexterity and expertise, used to measure task proficiency while teleoperating robotic platforms (Dulan et al., 2012b). To compensate the lack of force-feedback, two methods were implemented to provide sensory substitution in the form of color graphs and sound cues.

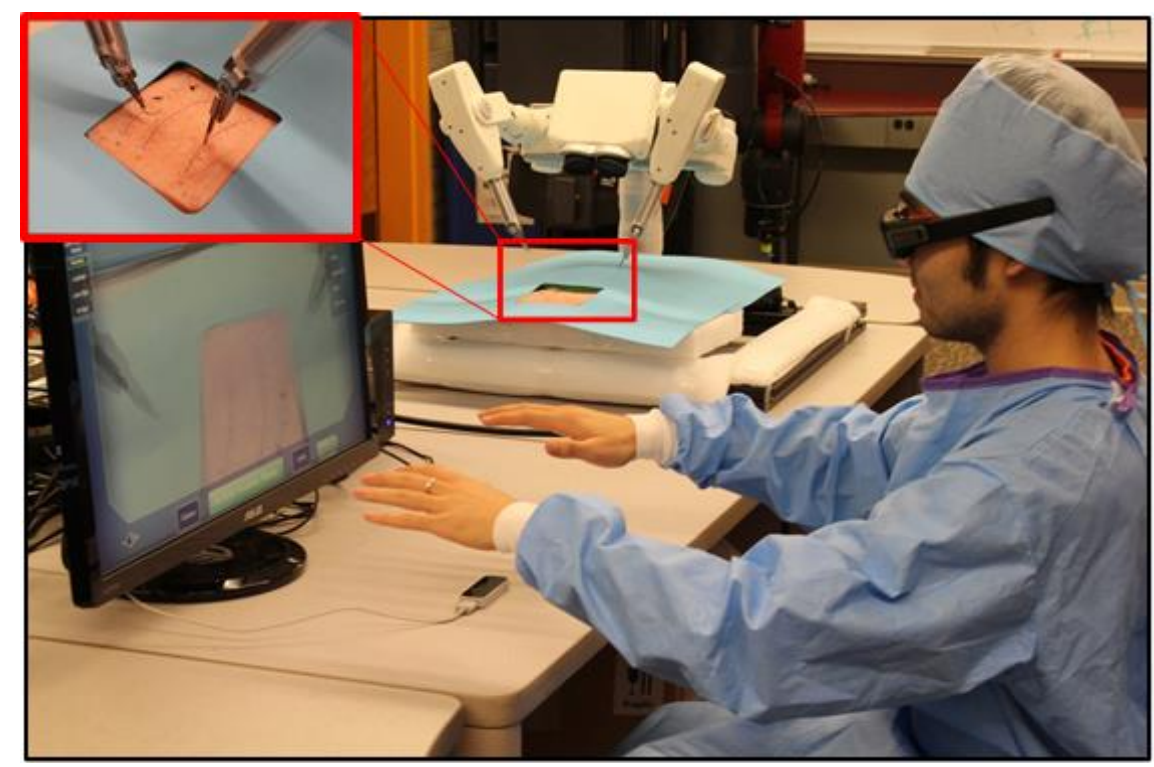

Figure 1. The user seated at the master console of the Taurus robot-assisted surgery system. The Leap Motion sensor placed below can track two hands for controlling the position and orientation of each end effector. Inset shows a custom fixture for a scalpel mounted on the Taurus end-effector in left arm, and in the right, surgical tweezers.

The main contributions of this paper are as follows: (1) the design and development of a simple touchless interface for telesurgery that allows the users to perform surgically related tasks with natural hand movements; (2) to determine whether a touchless type of interface can achieve a comparable performance to touch-based interfaces for robotic surgery; and (3) to evaluate the impact of sensory substitution while using touchless interfaces for surgical tasks.

The outline of the rest of this paper is as follows: The first section reviews the state of the art in telerobotic surgery and gestural interfaces, followed by an overview of the implemented system. The next section contains all pertinent information about the first set of experiments and is where we compared the performance of five different interfaces to perform two surgically related tasks; different objective and subjective (usability related) metrics were selected. For the second set of experiments, two of the previous five interfaces were selected under specific criteria to further compare the performance of touch-based against touchless interfaces; once again several metrics 
were measured and analyzed. To finalize the work, a section discussing findings is presented together with conclusions.

\section{Background}

\subsection{Telerobotic Surgery}

Given its dexterity, precision, high resolution, accurate motion planning, and execution capabilities, telerobotic surgery has become a gold-standard in several types of surgical procedures and outperforms traditional minimally invasive surgery, which relies on encumbered laparoscopic control. The epitome of the current paradigm is the widely adopted, commercial da Vinci robot (da Vinci Surgery, 2015). In that scheme, a surgeon manipulates a 7-DOF input controller, while mechanical linkages are used to stabilize and project those movements to the patient's site; there, a slave station drives robotic arms, including cameras and surgical tools that mimic the surgeon's hand movements with its end-effectors' motions. The surgeon's console includes a set of controls similar to the Omega haptic device (Force Dimension, 2015) but without haptic information, thus limiting the tactile sensorial information available to the surgeon. However, this form of control limits the range of manual operation, compared to in-situ surgery (Talamini, Campbell, \& Stanfield, 2002). In terms of the cognitive load, the surgeon is required map his/her natural hand gestures used in in-situ surgery to directional commands. This may result in unnecessary delays and cognitive load (Carswell, Clarke, \& Seales, 2005). Further, it can generate a sense of detachment (Sengül et al., 2012) and may increase the risk of breaking sterility (Hartmann et al., 2004; Jacob, Li, \& Wachs, 2011).

Similar forms of controls have been applied to several other surgical systems for conducting surgical procedures with evident success (e.g., ZEUS, AESOP, Mazor, Raven II) when evaluated in surgical procedures (Beasley, 2012). However, using touchless interfaces, where the gestures used to control the robot resemble those used in direct surgery, may have additional benefits that are worthy of exploration. One example would be cases where natural-hand operational dexterity associated with skillful in-situ surgery may be compromised (Rosen, Hannaford, \& Satava, 2011).

The use of the surgeon's bare hands allows for an increased mobility and natural expressiveness not found in the same form in standard-control consoles (Rautaray \& Agrawal, 2012; von Hardenberg \& Bérard, 2001). While touchless interfaces offer an attractive alternative solution to the problems mentioned, there is no straight-forward method to deliver tactile sensation. A potential parital solution is found in the sensorial substitution research area (Bach-yRita \& Kercel, 2003), where feedback is provided to the user by complementary modalities. For example, the visual system can be used to provide information related to skin contact, depth of incision, or tissue manipulation in a surgical teleoperation setting (Hoshi, Takahashi, Nakatsuma, \& Shinoda, 2009; Sodhi, Poupyrev, Glisson, \& Israr, 2013).

\subsection{Gestural Interfaces}

Innovative methods for interaction have become available for numerous applications with advancing technology. Although traditional interfaces such as mice and keyboards have grown to be familiar, they limit the naturalness with which users can interact with computer systems and have repercussions on the overall speed of task completion. For the last two decades, efforts have been made to incorporate hand gestures into the field of Human Computer Interaction (HCI) (Pavlovic, Sharma, \& Huang, 1997). Among prospective solutions, there are two mainly different approaches: one based on glove-based interaction and another based on video-based noncontact interaction.

The focus of this work will move toward the second alternative, since the first one would be trading a cumbersome mechanical interface for another one, in the form of a glove. Analog efforts 
have been observed in the development of new gaming interfaces to enhance the users' overall experience (Carrino, Ridi, Mugellini, Khaled, \& Ingold, 2012; Jakus, Guna, Tomažič, \& Sodnik, 2014; Oikonomidis, Kyriazis, \& Argyros, 2011; Weichert, Bachmann, Rudak, \& Fisseler, 2013), which have also impacted the field of HCI and gesture recognition. These innovations include Leap Motion (2015) and Kinect or electromyography (EMG) armbands, such as the Myo armband (Myo, 2015).

Using computer vision techniques to recognize gestures, specifically hand gestures, is a widely adopted methodology in many fields, including assistive technologies for healthcare (Huang, 2011; Monnich, Nicolai, Beyl, Raczkowsky, \& Worn, 2011; Wachs, Kölsch, Stern, \& Edan, 2011). The previously mentioned gaming technologies have also ventured into the field of robotic manipulation, particularly in surgical settings (Kim, Leonard, et al., 2014; Zubrycki \& Granosik, 2014).

\section{System Overview}

\subsection{Architecture}

A platform was developed throughout this project, which included touchless and touch-based interfaces, to compare objective and subjective advantages of each interface type in the context of surgical tasks. The developed platform allows substituting either type of interface seamlessly while maintaining the control protocol, feedback, and flexibility, independent of interaction form.

The telerobotic system was implemented on the Taurus robot. Taurus is a high fidelity telemanipulation robot with a 3D, HD display developed by SRI (Menlo Park, CA). It has two dexterous and compact arms that can be applied to the surgical setting; it enables surgical-like precision in a modular, portable frame. As shown in Fig. 2, it has two independently controlled manipulators with 7-DOFs each. The left end-effector includes a custom fixture for mounting tools, like a scalpel, while the right one functions as surgical tweezers.

Real-time visual feedback information is provided using a stereoscopic camera system and presented to the user in a 3D stereo display. The left and right views are accurately integrated using Nvidia's stereoscopic glasses, 3D Vision 2.

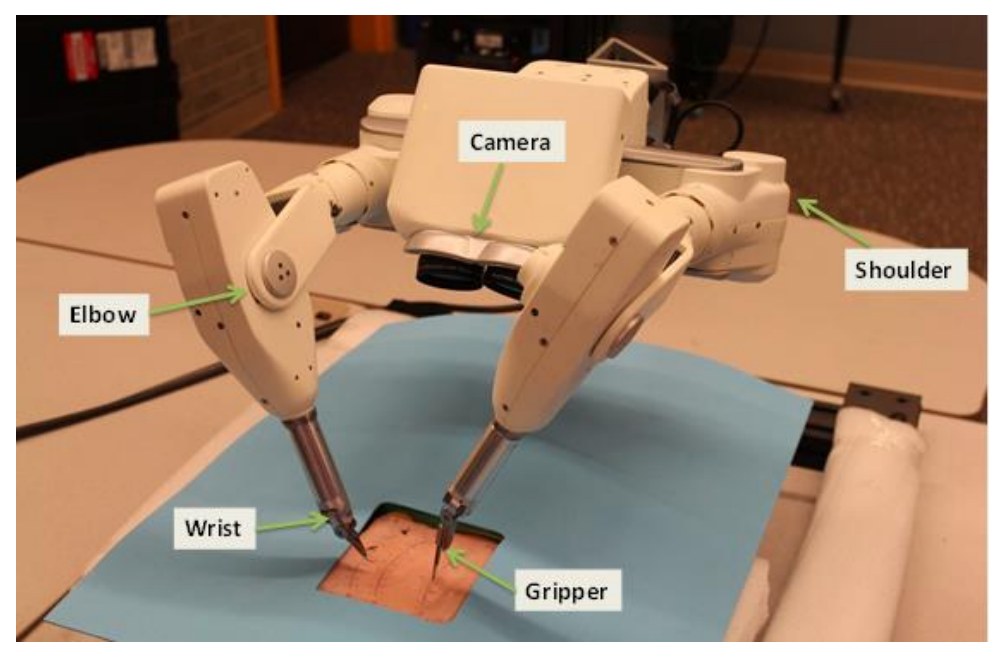

Figure 2. Picture of Taurus with a scalpel mounted on the left gripper. 
The system architecture is presented in Fig. 3. Using one of the five interfaces available, the user performs gestures to control the Taurus' navigation to complete a given task. These gestures are part of a lexicon that maps specific instructions to gestures through image processing, segmentation, and registration processes, which will be discussed later. Once the gesture is recognized, a motion trajectory is generated to replicate the user's motion in the robot space through 3D projection and coordinate mapping of the user space. Subsequently, the trajectory is converted to robotic commands using the Robot Control Scheme module. While performing the task, the user is able to receive feedback from Taurus by both visual and sound cues. Using the stereo cameras mounted on the robot, a 3D image is generated and presented to the user, as well as information regarding stiffness, position, and orientation of the tooltips. This information is presented to the user through color and sound feedback modalities or by direct force on the controllers. Based on this feedback, the user generates a subsequent set of commands to complete the task.

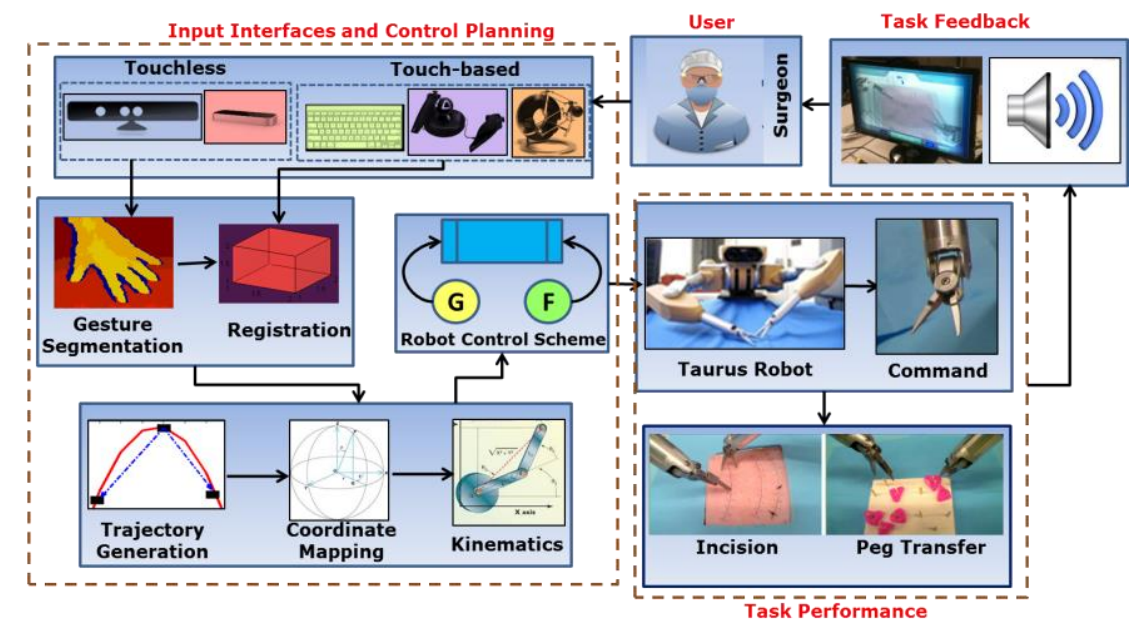

Figure 3. System Architecture.

In the following subsections, details will be provided about design rationale behind each interface.

\subsection{Interface Design Rationale}

To enable human teleoperation, both a control interface and supplementary control protocols were developed in the selected platform, and they are described below. Situation awareness of the remote environment is enabled through a feedback mechanism, which relies on visual, acoustic, and force-feedback (the latter in the case of the touch-based interface). The control functionally is provided by physical motions; perception is provided through visual displays, acoustic cues, and contact forces (for the touch-based conditions). The specific interfaces and forms providing necessary levels of control and perception are determined based on available state-of-the-art technology.

\subsubsection{Mapping Interfaces to Actions}

Five control interfaces were selected for this comparative study, among which there are three touch-based and two touchless. The touch-based interfaces included: a regular keyboard, a Hydra gaming controller (Razer Inc., 2015), and a Omega 7 haptic device (Force Dimension, 2015). A Leap Motion controller (Leap Motion, 2015) and a Kinect (2015) were used as the touchless interfaces. A picture of all interfaces is shown in Fig. 4. 


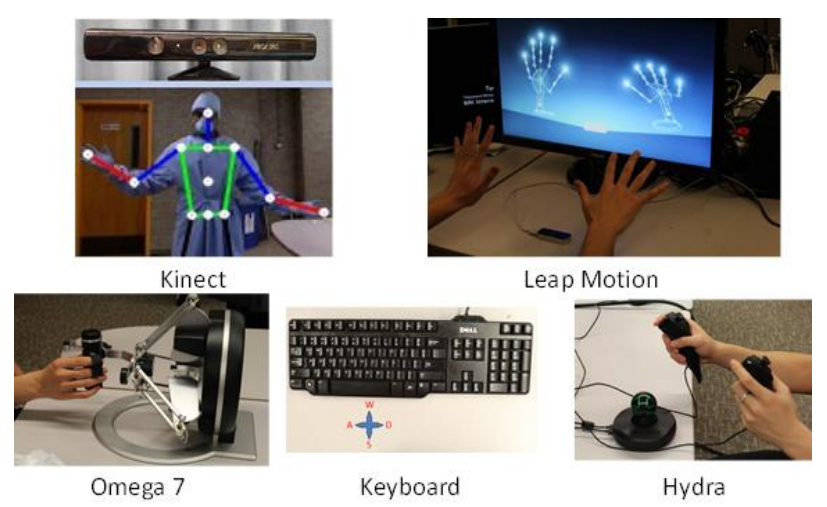

Figure 4. Five different interfaces to control Taurus: touch-based and touchless among them.

Aside from selected interface, a foot pedal was used as supplementary control input along with every interface to toggle the engage/disengage servo mechanism, which helped the operators switch the working space or stay idle. The foot pedal has two switches and can provide 2-DOF toggle control. The frequency of foot pedal pressing indicates the frequency of working space shifting during the experiment, and such interruptions during complex and cognitively demanding tasks are a key indicator of the operator's engagement level (Oviatt, 2006). The raw data gathered from the sensors was filtered using a low-pass filter to cancel noise variation caused by the control input modalities.

The Kinect teleoperation control modality is based on tracking the user's body movements. Tracking is accomplished using an algorithm included in the MS-Kinect SDK that fits a skeleton model to the user's 3D view. This results in a continuous stream of body joints' and hands' positioning. Taurus is controlled by the hands' trajectories, after applying a sliding window mean filter (with a length of five frames) to cancel both the operator's unintentional hand tremor and the noise associated with the optical sensor. Once the operator begins a surgical task, the initial position becomes the origin of the user space; and all subsequent movements are obtained relative to that initial position. These movements, in turn, are tracked and converted to movement control signals. When the hand motion magnitude is above a predefined threshold $\tau$, a motion instruction is sent to the robot. The direction that exhibits the largest movement is chosen as the user's intentional moving direction. Then, the distance between the current hand position and the user's space origin determines movement speed in this direction. The farther away the hand is from the origin, the faster the robot arm moves. The user's hand can move up/down, left/right, and forward/backward, thus providing 3-DOF control.

We also leveraged the foot pedals to provide the remaining DOFs' control. Whenever the left pedal is pressed, the robot is in a position-moving mode, and the 3-DOF control from user's hand will drive the translational movements of the robot. When the right pedal is pressed, the robot is in an orientation-moving mode, and the 3-DOF control will change the orientation pose of the endeffector. When both pedals are pressed, the up/down motion is mapped to the open/close motion of the gripper. In total, with the help of the foot pedals and the hands' 3-DOF control, the user can control all of the robot's DOFs. Algorithm 1, shown below, describes the method described. A direct mapping from hand-position to tooltip-position was not appropriate for the Kinect control protocol, because position sensing accuracy is not precise enough for fine movement. That kind of mapping would result in erroneous robot movements. Alternatively, different control protocol designs for Kinect have been used (DeSouza, Jiang, Wachs, \& Duerstock, 2014; Jacob \& Wachs, 2014) that entail using the hand's relative movement with respect to a reference frame. 


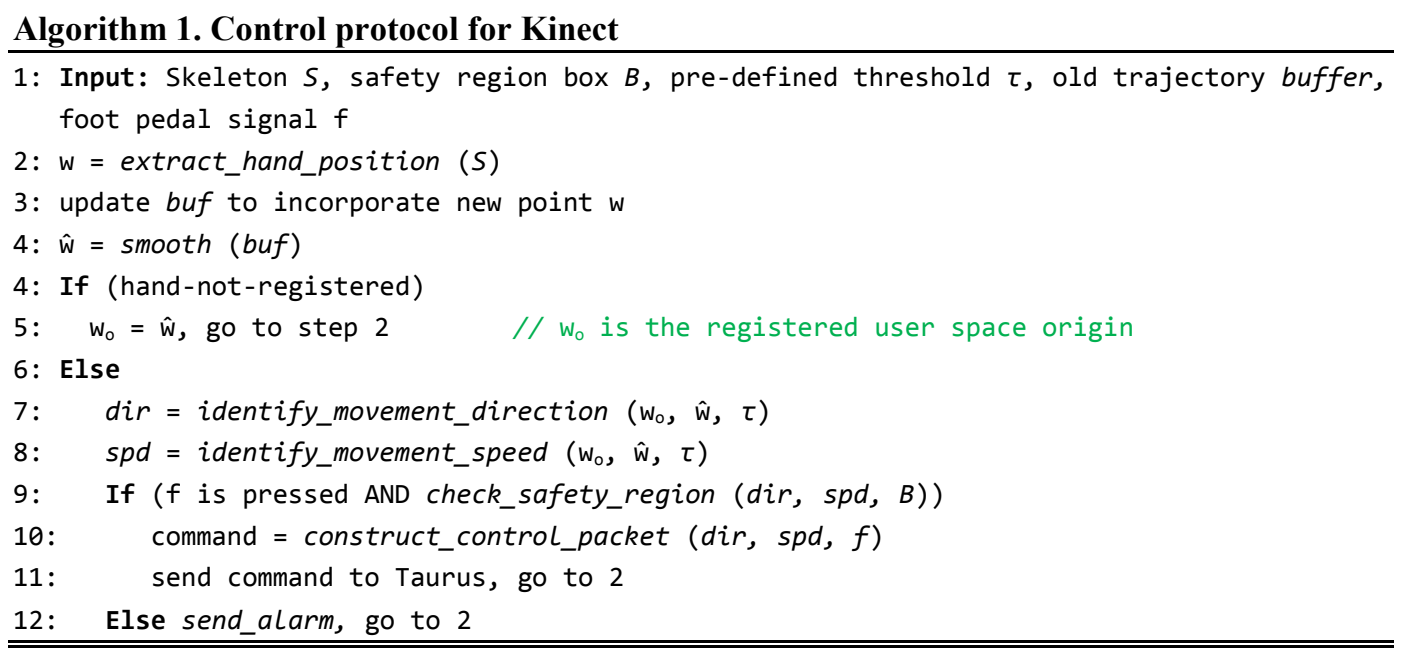

Leap Motion is a desktop infrared sensor that allows hand tracking and hand gesture recognition in real time. Leap Motion SDK was used to obtain the roll, pitch, and yaw angles' magnitudes, based on the position and direction of the hands. The center of the palm $(x, y, z)$ was mapped to correspond to the position of Taurus' end-effector. The orientation of each palm (roll, pitch, and yaw) was mapped to control the orientation of the Taurus' corresponding endeffector. The pinch motion between the thumb and the index finger was used to control the open/close action of the gripper. This is the same gesture humans naturally perform to pick up a delicate object with their hands.

Omega 7 is a haptic interface with a 7-DOF that delivers force-feedback reflecting contact forces at a rate of $4 \mathrm{KHz}$. From this device, forces can be applied to the human hand for reflecting forces experienced by the robot's tooltip. Omega 7 working space maps the exact position and orientation of both end-effectors; opening and closing of the robot's tooltip was controlled through a pinching motion between the thumb and index fingers.

Hydra is a control interface often used for gaming. The 3D controls can generate user hands' exact location and orientation by relying on magnetic motion sensing.

For a keyboard to be used as the main input modality, specific keystrokes were assigned to motion control commands. One set of keys was allocated for moving the end-effector in one particular direction, and a different set controlled the roll, pitch, and yaw angles of the endeffector. While this type of interface was not as intuitive as others, it allowed precise control.

Fig. 5 lists the mapping between robot movements and user control for all five interfaces, for the left arm. Each robotic arm has three DOFs for position control, three DOFs for orientation control, and one DOF for gripper control. Similar configurations were used for rightarm control. The user is using both hands along with the foot pedal to deliver 7-DOF control from each hand. The letter " $L$ " indicates the left pedal pressed down, and " $R$ " is for right pedal pressed down.

\subsubsection{Coordinate Registration Process}

The registration process is a prerequisite of interface operation to retrieve parameters once the interface is selected. It consists on mapping the reference frame in which the user works with the frame associated with the task space. In the beginning of teleoperation, the first 10 control input coordinates are recorded, and an origin of the user space is generated from their running average. This origin of the user space is then mapped to the origin of the robot space. 




Figure 5. Mapping between robot movement (left arm) and interface control design.

During teleoperation, the operator's movements, relative to this origin, are then interpreted as practical control instructions. This process applies to all interfaces other than the keyboard, since the latter does not use 3D coordinates to evoke control motions; instead, keystrokes are used. The registration process takes place whenever the foot pedal is reactivated by the user. Since the user starts teleoperation not always for the same exact position, the origin of the user's coordinate frame must be aligned with the robot's coordinate frame first, before proceeding with the surgical task.

\subsubsection{Safety Operation and Region Determination}

Virtual bounding boxes were implemented around both the user and task space in order to ensure safe motion during robot teleoperation. In the user space, a virtual rectangular region is created around the user' hands to maintain the input coordinates within a permissible operational range. Working within this region also assures that the hands are within the optical sensors' field of view. Analogously, a similar region is established around the robot at the task space. This region limits the outer reach of the robot and assures that the robot's movements are maintained within the operational range. The robot's working space is divided in two regions, as shown in Fig. 6, allowing each arm to move only in its half with some overlapping in the middle. This assures that the robot's forearms do not collide with the camera. When bounding box limits are reached, the user is notified by a beep sound.

\subsubsection{Feedback Generation}

Force-feedback is critical in robot-assistive surgery, since the surgeon needs to perceive force exhorted on the patient (Wagner, Stylopoulos, Jackson, \& Howe, 2007). To that end, a force 


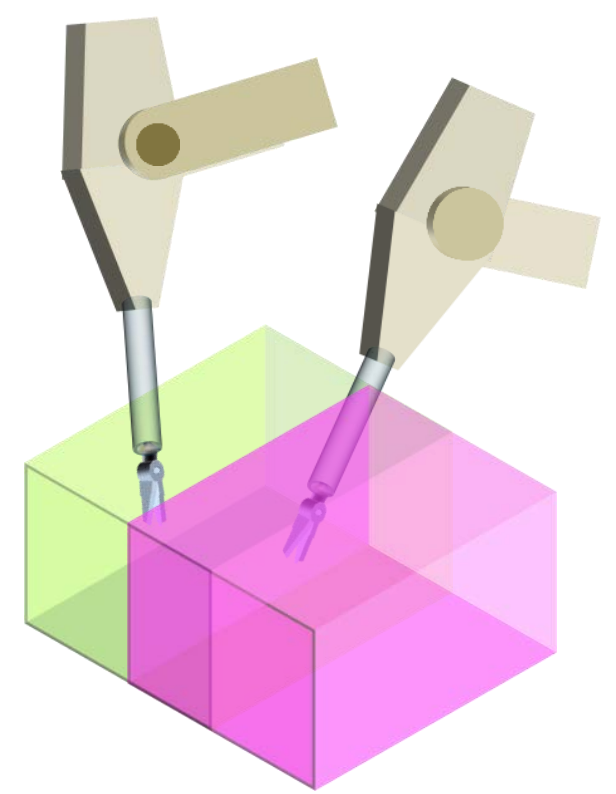

Figure 6. Taurus' operation space. The left gripper is allowed to work only in the pink region and the right gripper in the green region. Both grippers can reach the overlapping area in the middle.

sensing algorithm and force-feedback mechanism is developed on our system. The first step is to measure the force that the end-effector is experiencing, and the second step is to convey such information back to the user in a promising manner.

The force on the tooltip is sensed based on the difference between the command position and the robot's reported position. A large difference occurs when the end-effector directly contacts an object. It responds according to a conventional spring model, where the sensed force is linearly proportional to the difference between the command position and the response position. Details are illustrated in Algorithm 2.

After the force has been measured using the algorithm above, it needs to be relayed back to the user accordingly. As mentioned in previously, one of the drawbacks of working with

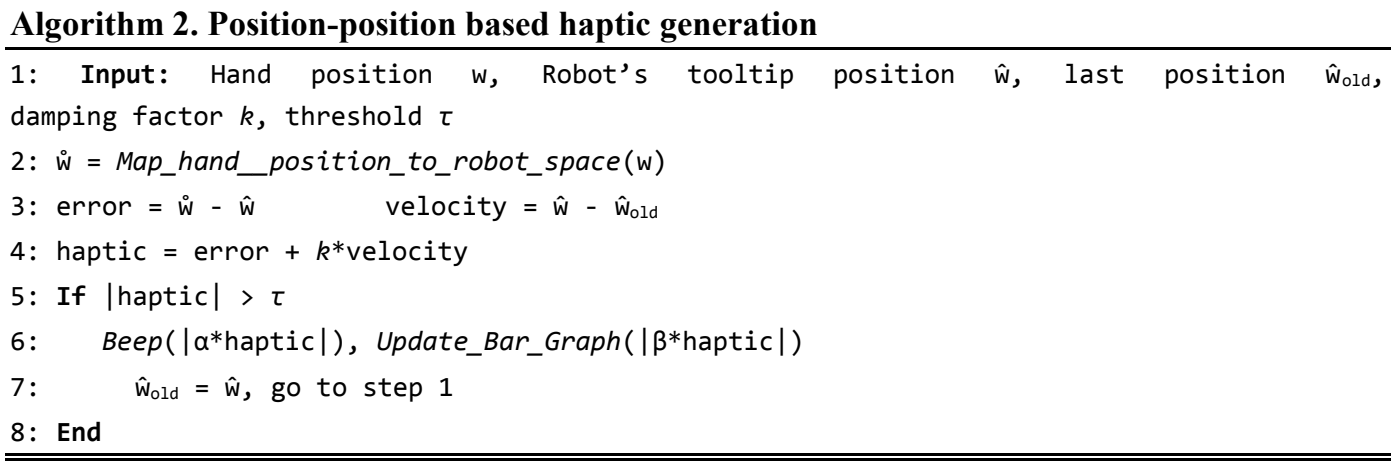




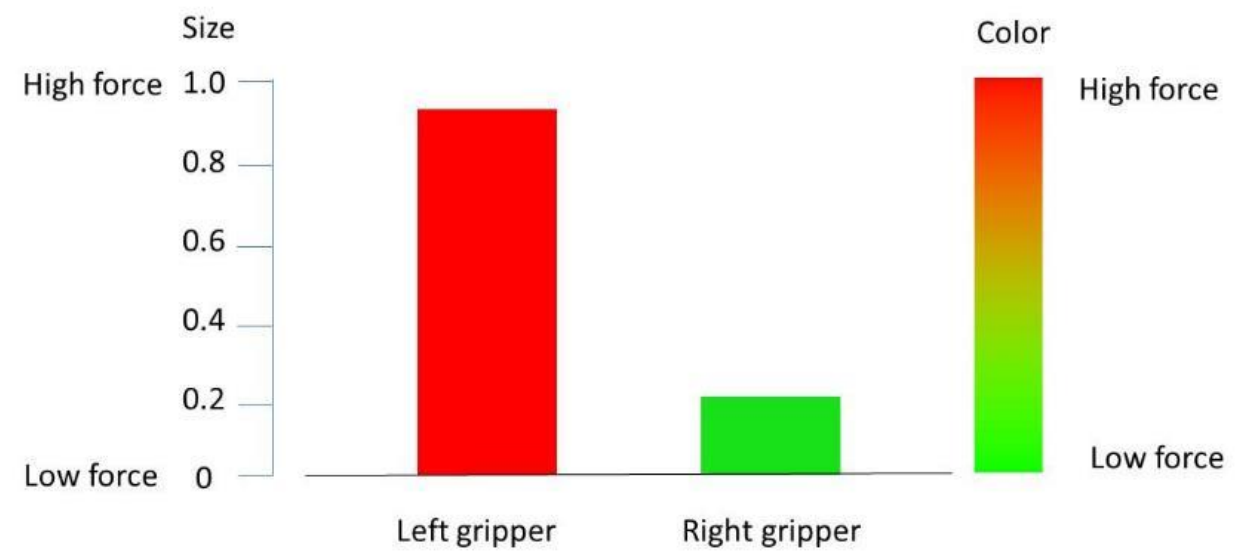

Figure 7. Example of the bar graph for sensory substitution. The left and right bars indicate the force of the left and right gripper, respectively. The size and color of the two bars change according to the amount of force exerted to each gripper.

noncontact hand gestures is the lack of force-feedback. This problem is partially addressed using sensory substitution techniques, based on vision and audio cues.

The operator receives physical contact information about the force exerted at the tooltip through two bar graphs; each bar indicates the force of one tooltip, as shown in Fig. 7. The size and color of each bar changes proportionally with the amount of force applied to the end-effector. At the same time, a beep sound is generated with its pitch modulated according to the force's magnitude. Both acoustic and visual channels are meant to increase the user's awareness while performing the surgical task.

The sensory substitution technique is used for all five interfaces in the first two experiments. While Omega can provide force-feedback, this option was disabled to allow a fair comparison among all the control modes. In the last experiment, we compared characteristics of the touchless and touch-based interfaces, including their functional advantages. In these experimental scenarios, Omega's force-feedback functionality was enabled.

\section{Experiments 1 and 2: Incision and Peg Transfer}

In this section, two different experiments are described and analyzed, with specific goals and metrics evaluating the performance of five different interfaces, among which are touch-based and touchless. Both experiments are surgically related tasks used for training medical students as found in the literature, and the layout is shown in Fig. 8 (Dulan et al., 2012a; Nielsen, Störring, Moeslund, \& Granum, 2004). The main hypothesis that was tested throughout the experiments was that touchless interfaces would lead to better performances compared to touch-based ones, with respect to task efficiency, failure rate, and naturalness of movement. This hypothesis was deduced from the fact that touchless interfaces would provide higher level of naturalness and freedom of motion, enabling surgeons to perform gestures similar to real gestures used in surgery. Intuitively, these are clear benefits to robotic telesurgery. The detailed hypothesis for each conducted experiment will be introduced in each section respectively, under the context of the task and the specific evaluation metrics. 

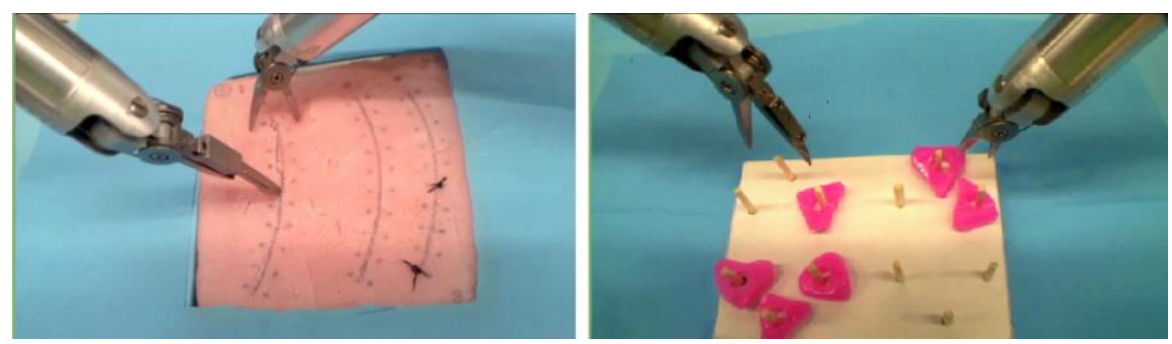

Figure 8. Left image: Incision task; the right gripper holds the surgical pad while the scalpel on the left gripper makes the incision, following the target curve. Right image: Peg transfer task; the left gripper picks up a pink peg from a pole on the left side of the board, passes it to the right gripper, which in turn, places it through a pole on the right side of the board, following a specified order.

\subsection{Incision Experiment}

In this experiment, participants were required to teleoperate Taurus to conduct a curved incision, following a target incision line predefined in 2D and marked on a surgical pad, as shown in Fig. 8 (left). The operator was asked to keep a fixed depth during the incision, and at the same time, follow the target curve as closely as possible. Fig. 9 shows five experimental traces made by one subject using Omega 7. The operator was instructed to cut through the incision until haptic feedback (in the form of a visual colored bar graph) and a modulated beep sound was heard. This value represented the end of the epidermis layer. Performance in this experiment was determined through the distance from target annotation and the control variance.
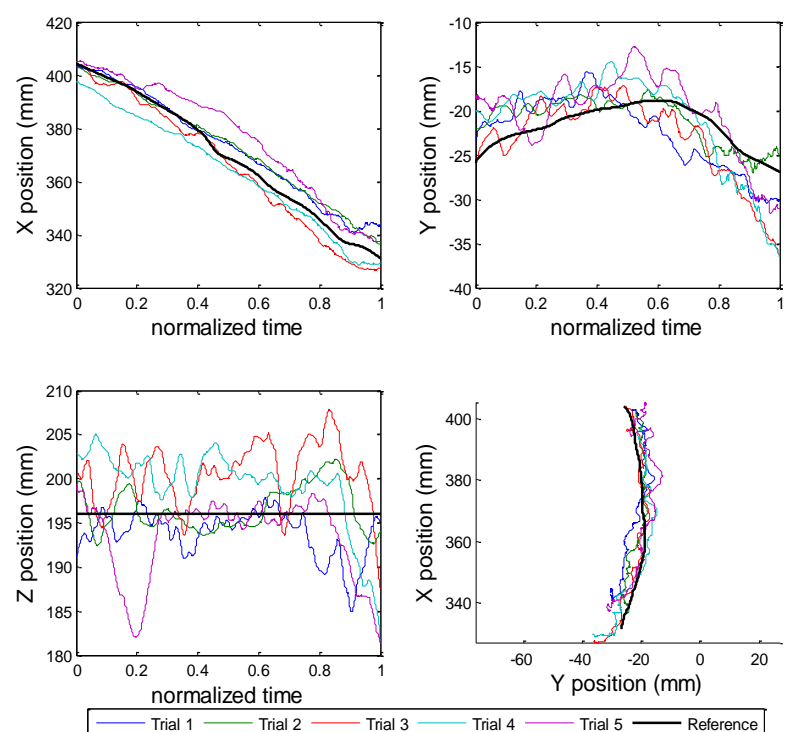

Figure 9. Experimental trajectories across five trials from one participant. Upper left, upper right, and lower left figures show trajectories along $\mathrm{x}, \mathrm{y}$, and $z$ axis versus normalized time. The lower-right diagram shows an $x-y$ plane projection. Black lines indicate the reference trajectory. 


\subsubsection{Methodology}

Different metrics were used to measure the user's performance in this task, mainly from the control-precision perspective and control-stability perspective.

To assess control precision, we measured the deviation error between the user's performed incision curve and the reference curve (the tooltip orientation error was not considered). Twenty equally spaced landmark points on the target trajectory were chosen to calculate the distance between the experimental curve and the reference one. Each landmark was used to obtain the normal direction to the reference annotation. The intersection between the normal to the landmark and the actual trajectory was used to measure the point-wise error. A zoom-in segment of both trajectories, showing the aforementioned error metric, can be seen in Fig. 10.

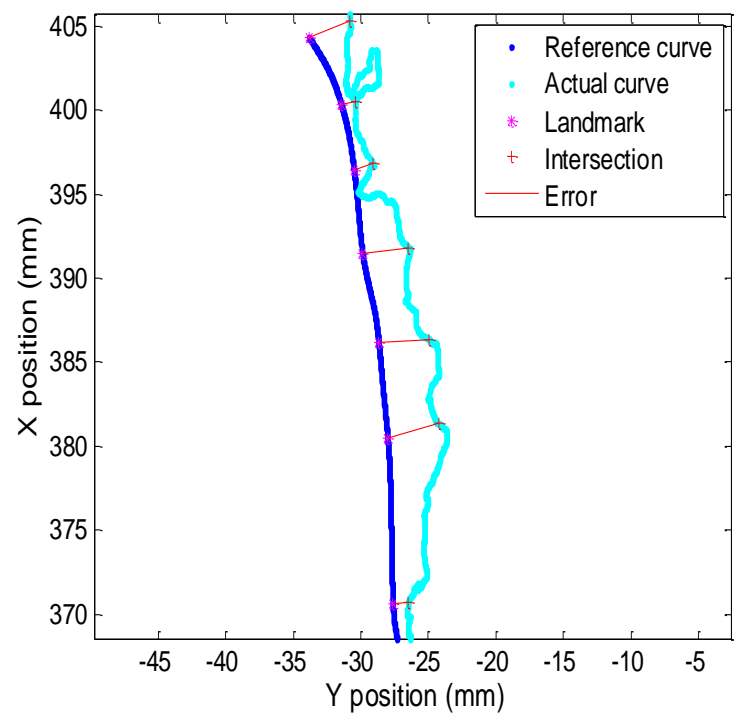

Figure 10. A zoomed-in inspection of the reference trajectory (blue) and an experimental trajectory (cyan) on the $x-y$ plane. The red lines are perpendicular to the reference's tangent at the landmark point and intersect the actual trajectory; the length of the red line indicates the discrepancy between experimental trajectory and the reference at this landmark.

To evaluate control stability, we analyzed depth fluctuation of the actual trajectory that the user performs. The root mean square (RMS) of the distance between the depth trajectory and its time average was used to quantize the depth fluctuation, as shown in the following equation:

$$
\text { Depth Fluctuation }=\sqrt{\frac{1}{N} \sum_{i=1}^{N}\left(X_{i}-\bar{X}\right)^{2}}
$$

where $X_{i}$ - Depth trajectory of one trial.

$N$ - Length of the trajectory.

$\bar{X}$ - Mean depth trajectory.

While participants were required to conduct the exact same task five times, no trajectory was identical to another. The number of joints and degrees of freedom in the human arm, shoulder, and torso allowed the operator to adopt multiple body configurations for reaching a single $\mathrm{x}, \mathrm{y}$, and $\mathrm{z}$ 
position. This redundancy is both beneficial and problematic. On one hand, it brings flexibility for the participants faced with obstacles, but it also creates an ill-posed control problem to be resolved by the human motor system (Nisky, Hsieh, \& Okamura, 2013). To understand the role of human motor control redundancy and the role it played across the five implemented interfaces, structured variability was computed using Eq. (2) (Nisky et al., 2013).

$$
V[t]=\sum_{i=1}^{N}\left(w_{i}[t]-\bar{w}[t]\right)^{2} d^{-1} N^{-1}
$$

where $\quad w$-Task space vector, in $3 \mathrm{D}$ space this is a vector of dimension $R^{3}$

$\bar{w}$ - Task space vector average across trials as the reference trajectory

$d$ - Number of DOFs in the task space, corresponding to the dimension of $w$

$N$ - Number of trials to be averaged

This structured variability measures the stability of a variable around a time-varying reference trajectory under perturbation. A naïve interpretation of this metric is that an operator has more control freedom through the teleoperation task with higher structured variability. More control freedom might point at a more natural interaction, or alternatively, unprotected large movements.

\subsubsection{Experimental Design}

The experiment design followed a five-treatment/two-period balanced crossover design pattern (Carriere \& Reinsel, 1992; Wallenstein \& Fisher, 1977). This experiment involved the participation of 10 students, 5 males and 5 females with an average age of $30.5(S D=6.3)$. Each subject was assigned to teleoperate Taurus using 2 out of the 5 available interfaces through the task, for 5 repetitions each interface, thus resulting in a 5 treatment $/ 2$ period design. Each subject tried two interfaces in a sequence (hence the crossover design), and the order of the two interfaces was randomized to compensate for task learning. Under such scenario, there were 20 repetitive measurements for each interface, and all 10 combinations of 2 interfaces ( 2 chosen from 5) were tested by the same number of participants, resulting in a balanced experimental design.

The underlying hypothesis tested in this experiment was that touchless interfaces should lead to a higher level of task performance (measured through deviation error and depth fluctuation) and control freedom (measured through structured variability) compared to touchbased ones. To assess that hypothesis, an ANOVA was conducted after the experiments were finished and metrics were recorded. The null hypothesis in this analysis states that all five interfaces exhibit the same level of performance with respect to each metric (deviation error, depth fluctuation, and structured variability, etc.). The independent variable (IV) was the interface used, and the dependent variables (DVs) were the different measures/metrics values. To reduce the potential random effects brought by any individual difference, subject identity was treated as a random effect in the ANOVA model, so that the test result can be related to the interface difference. After the ANOVA was calculated, a Games-Howell post-hoc test was implemented to inspect any pair-wise difference. Overall, through ANOVA and the post-hoc analysis, we were able to compare the DVs to check if there were any intrinsic differences between the IVs.

Preliminary results were used to estimate the required population size to calculate all the metrics with necessary significance. Under such a priori information, a sample size of 20 observations can generate a power larger than 0.99 . Visual feedback was available for the $\mathrm{x}-\mathrm{y}$ plane corrections, while feedback for the $\mathrm{z}$ plane (or depth) was offered in the form of acoustics and vision. The experiment was approved by Institutional Review Board (IRB), protocol number \#1408015151. 


\subsubsection{Results}

The deviation error from the target trajectory for each interface is shown in Fig. 11. Each bar represents the average and represents the $95 \%$ confidence interval for incision deviation distance for each interface. Levene's test revealed that the homogeneity of variances between groups was violated, thus a Welch's ANOVA test was conducted and statistical significance was found $(p<$ 0.001). Games-Howell post-hoc test indicated that the Hydra and Kinect interfaces (group A) showed a larger deviation as compared to a keyboard, Leap Motion, and Omega (group B), with statistical significance $(p<0.001)$.

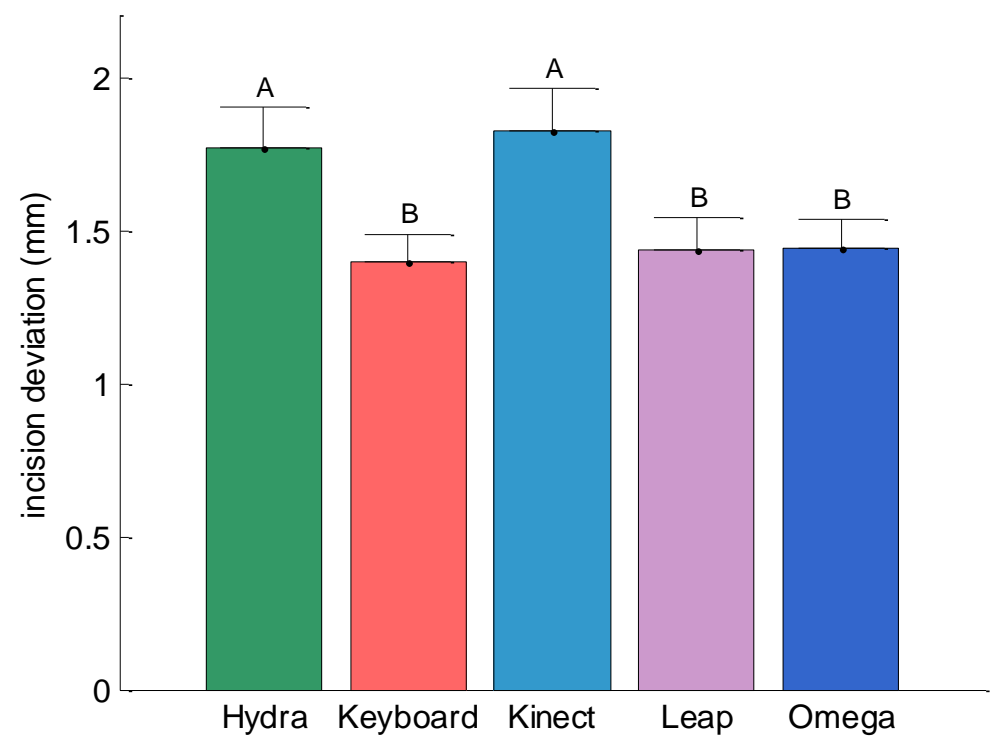

Figure 11. Deviation from the established incision path (in $\mathrm{mm}$ ). Interfaces with different letter indicate that there is a significant difference between them. (Welch ANOVA, $\mathrm{p}<0.001$; Games-Howell post hoc test, $\mathrm{p}<0.001$ ).

The depth fluctuation was calculated for all the trials of each interface, and the corresponding boxplots are shown in Fig. 12. The keyboard and Kinect showed the least amount of depth fluctuation among all the interfaces with statistical significance $(p<0.001)$. For both interfaces, the movement control in all axes are decoupled from each other, which facilitated maintaining a fixed depth during the incision task.

The result of the structured variance analysis applied to the experimental data is shown in Fig. 13. To compare the variance per DOF among different interfaces, we calculated the root mean square (RMS) value of the variance over time. Leap Motion showed the largest RMS value (44.5), which indicated that the user had a larger variability of movements. The second, in order, was Omega (23.3), followed by Kinect (15.2), Hydra (13.7), and keyboard (7.6). Using the keyboard requires constrained movements (as key locations are fixed), and therefore, the structured variability is low (i.e., lower control freedom).

\subsection{Peg Transfer Experiment}

The second task involved completing an abbreviated version of the FLS's Peg Transfer task, as shown in Fig. 8 (right). The operator controlled Taurus to pick up a soft ring (the peg) with its left arm from the left side of the pegboard, pass the ring to the right arm, and release it on top of a pole 
Zhou et al., Comparative Study for Telerobotic Surgery

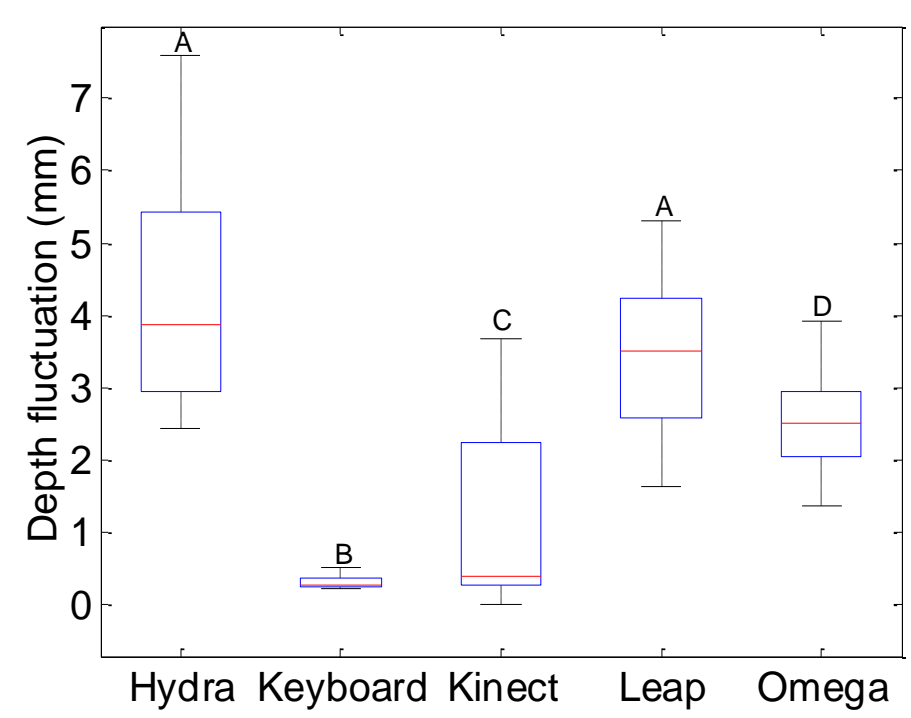

Figure 12. Boxplot of depth fluctuation across different interfaces. Different letters imply different means with significance. (Welch ANOVA, $p<0.001$; Games-Howell post hoc test, $p<0.001)$. The order from the smallest to largest depth fluctuation is as follows: Keyboard $<$ Kinect $<$ Omega $<$ Hydra $=$ Leap Motion.

on the right side of the pegboard. This process was repeated for three rings in a sequential order (the standard FLS Peg Transfer task requires moving six pegs). If the ring is dropped, the operator is allowed to pick up the ring and continue with the task. The number of total drops was recorded for further analysis. Best performance is achieved when the number of drops is minimalized, the learning rates are the steepest, and the task completion time is the shortest. Challenges associated

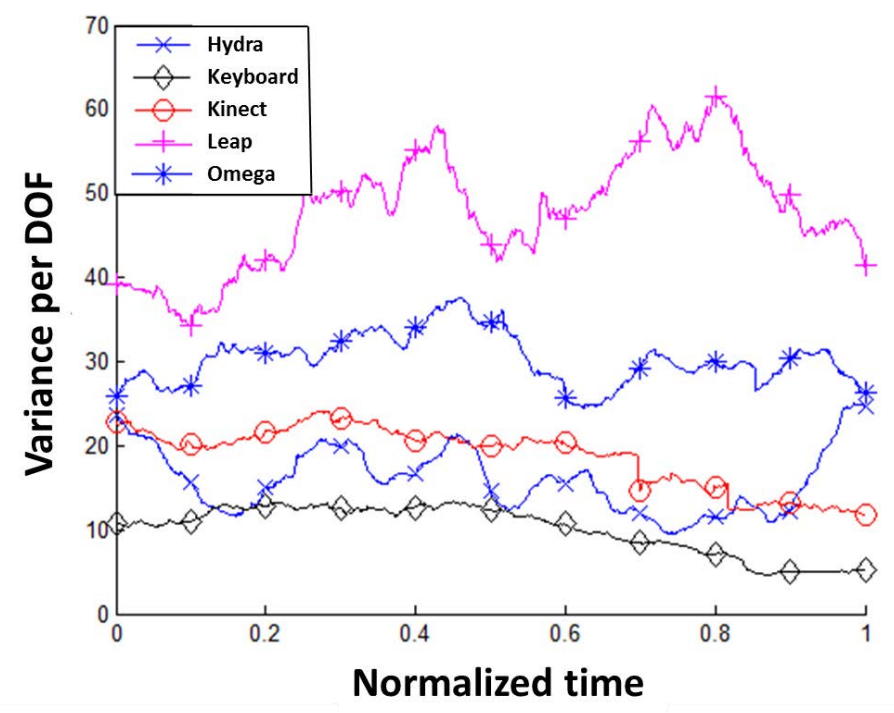

Figure 13. Trial-to-trial variability analysis as a function of normalized time averaged among different trials. The variance per DOF is shown for all five interfaces. 
with this task involve teleoperating both robotic arms simultaneously and cooperatively to transfer each peg from one gripper to the other effectively; it also requires high dexterity to move the tooltip without dropping the pegs and place them in the corresponding poles.

\subsubsection{Metrics}

Task completion time was the main metric analyzed in this experiment. Timing began when the user started moving the robot and ended when the user placed the last peg in the corresponding pole. The learning rate of task completion time over trials was calculated as follows. Firstly, a loglog regression was applied between the task completion time and the corresponding trial number, and then the exponential of the resultant slope of the fitted line was calculated as the learning rate, as shown below:

$$
\begin{aligned}
& a^{*}, b^{*}=\underset{a, b}{\arg \min } \| \ln (\text { Time })-a \ln (\text { TrialCount })-b \|^{2} \\
& \text { learning Rate }=\exp \left(a^{*} \ln (2)\right)
\end{aligned}
$$

A learning curve was later obtained based on the task completion time over trials and the learning rate calculated. Smaller learning rates indicate a faster learning process. The number of peg drops was also recorded as a metric and is an indicator of the level of two-hand coordination during teleoperation, since most peg drops occur when being passed from the left gripper to the right one.

\subsubsection{Experimental Design}

We used the same experimental design method as mentioned for the incision task, namely the 5 treatment $/ 2$ period balanced crossover design. The same participants for the incision task conducted the peg transfer experiment. The recorded metrics included task completion time and number of peg drops.

The underlying hypothesis tested in this experiment was that touchless interfaces should lead to better task performances (shorter task completion time and fewer peg drops) and faster learning rates. To assess that hypothesis, an ANOVA was conducted after the experiments were finished and metrics recorded. The null hypothesis in this analysis states that all five interfaces exhibit the same level of performance with respect to task completion time, number of drops, and learning rate. The independent variable (IV) is the interface used, and the dependent variables (DVs) are the different measurements. Same as the previous experiment, subject identity was treated as a random effect in the ANOVA model to reduce the potential random effects brought by any individual difference. After the ANOVA was conducted, a Games-Howell post-hoc test was implemented to inspect any pair-wise difference. Overall, through ANOVA and post-hoc analysis, we were able to compare the DVs to check if there were any intrinsic differences between the IVs.

\subsubsection{Results}

A learning curve was subsequently obtained to test the level of improvement with each trial and used to determine the learning rate for each interface, as shown in Fig. 14. The average task completion time, number of peg drops, and the learning rate are shown in Table 1. Experiment results revealed that the Hydra interface and Omega haptic controller led to shorter task completion times than the Kinect, the keyboard, and the Leap Motion interfaces, with a significant difference (Welch's ANOVA and Games-Howell post-hoc test, $p<0.001$ ). Different superscript letters indicate different group means with statistical significance. Results also indicate that overall the touchless interfaces have shown a faster learning rate than the touch-based ones $(r=69.86 \%$ for the Leap Motion and $r=85.42 \%$ for the Kinect). Assuming fixed learning rates for all five interfaces, the curve for the Leap Motion would be the first one to surpass the completion time for all other interfaces. This hints at the possibility of yielding the best performance with this touchless interface by having more practice with it. 
Zhou et al., Comparative Study for Telerobotic Surgery



Figure 14. Fitted learning curve for task completion times across all interfaces.

\subsection{Multi-Objective Problem}

To integrate the various metrics into a single and compact representation, a consistent measure was developed by ranking all the interfaces based on their performance in each individual metric. For each metric, smaller ranking values were assigned to preferred interfaces. The same number could be assigned to a bundle of interfaces if no statistically significant difference was found among them. For example, for the metric of incision deviation, there were three interfaces that were ranked the same (rank 1), and the two remaining interfaces were also ranked the same (but worse than the other three). This is denoted by the string 11144 . For learning rate, which is a pure number and shows no statistical nature, the relative distances between those numbers were used to determine the rank.

\subsubsection{Metrics and Ranking}

The overall ranking is shown in Fig. 15 as a radar map. It is indicated that there is no such an interface superior to all the others in all the metrics. Finding the best interface under this

Table 1. Average Performance for the Peg Transfer Task. Different superscript letters (A, B, C) indicate statistically significant different group means $(p<0.001)$ using the Games-Howell posthoc test.

\begin{tabular}{|c|c|c|c|}
\hline Interface & $\begin{array}{c}\text { Completion } \\
\text { Time } \pm \text { CI }(\mathbf{s})\end{array}$ & Peg Drops \pm CI & $\begin{array}{c}\text { Learning } \\
\text { Rate }\end{array}$ \\
\hline Hydra & $107.9^{\mathrm{A}} \pm 10.00$ & $0.2^{\mathrm{A}} \pm 0.2$ & $92.71 \%$ \\
\hline Keyboard & $271.9^{\mathrm{B}} \pm 37.33$ & $0.15^{\mathrm{A}} \pm 0.2$ & $93.83 \%$ \\
\hline Kinect & $594.7^{\mathrm{C}} \pm 64.64$ & $0.4^{\mathrm{B}} \pm 0.3$ & $85.42 \%$ \\
\hline Leap Motion & $360.2^{\mathrm{B}} \pm 70.98$ & $1.3^{\mathrm{B}} \pm 0.6$ & $69.86 \%$ \\
\hline Omega & $121.3^{\mathrm{A}} \pm 13.34$ & $0.2^{\mathrm{A}} \pm 0.2$ & $85.69 \%$ \\
\hline
\end{tabular}




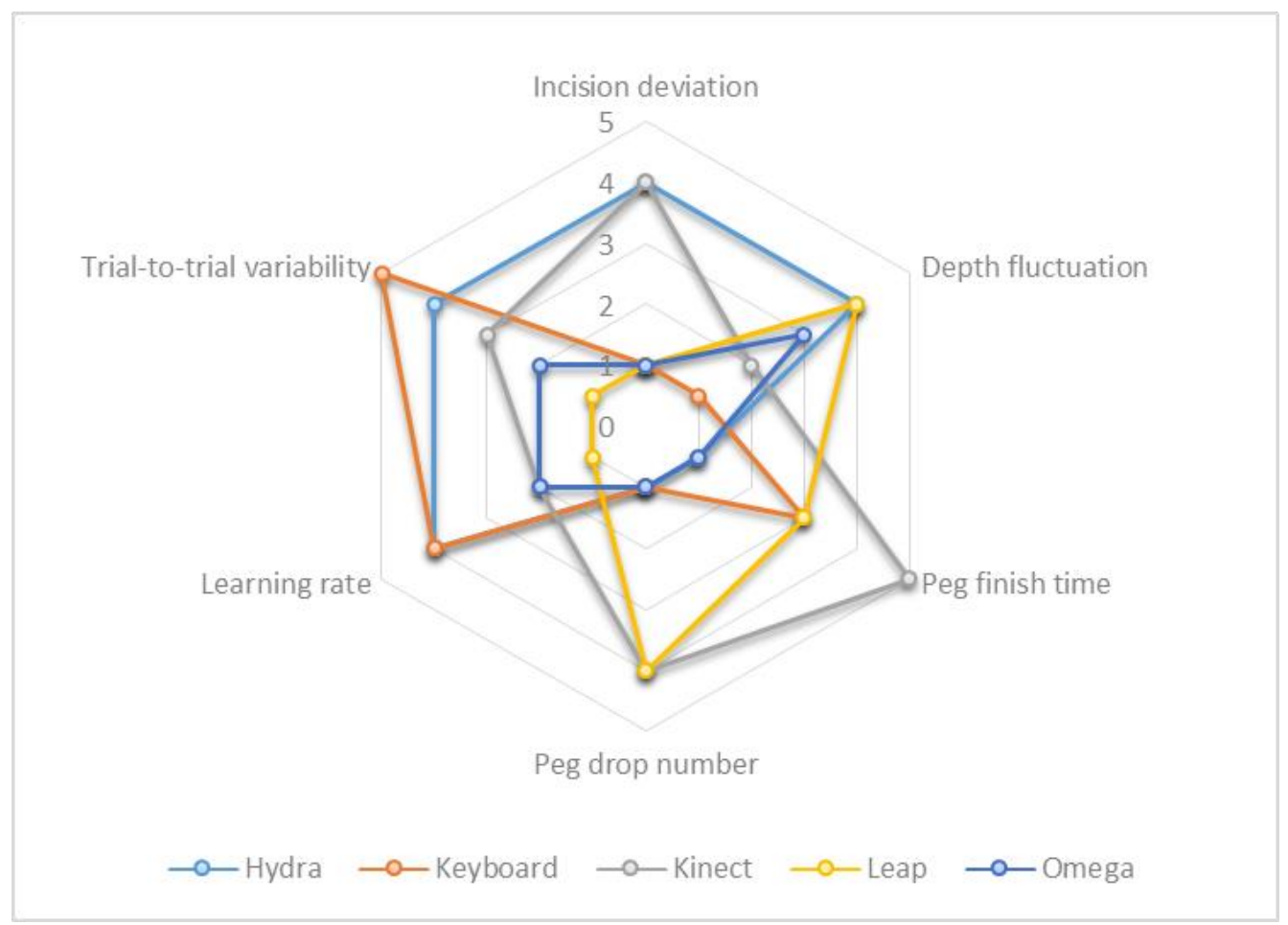

Figure 15. Radar map for the ranking of all interfaces (small numbers mean higher preference; same number means no statistically significant difference).

comprehensive ranking scenario is equivalent to solving a multi-objective optimization problem of finding the interface with the minimum ranking value over all criteria. Since some objectives are conflicting, we are interested in finding the Pareto front. The optimal Pareto front was calculated and included all the interfaces except Hydra, which is outperformed by Omega in all the perspectives (including equivalence).

\section{Experiment 3: Needle Threading Challenge}

To further evaluate the touchless interfaces along with the sensory substitution method, we conducted a second set of experiments, where we compared the top performer of the touchless and touch-based groups, respectively. Among the touchless interfaces, Leap Motion was selected as a representative interface, due to its advantageous performance over Kinect in the first experiment. Force-feedback was provided using sensory substitution in this case. For the touch-based interface, Omega was chosen, since it outperforms Hydra in all the metrics. Omega's feature for force-feedback was activated during this task.

In this experiment, participants were required to teleoperate Taurus to conduct a threading task, as shown in Fig. 17. Performance in this experiment was determined through the task completion time, number of needle drops, number of pedal presses, and learning rate. The number of times the user resets the coordinate frame (expressed by the times that the user presses the control foot pedal) indicates how often the subject shifted the working space during the experiment. This value was chosen as a metric, because the number of interruptions during complex and cognitively demanding tasks is a key indicator of system usability (Oviatt, 2006). 
The focus of this experiment is to give a more comprehensive comparison between touchless interfaces and touch-based interfaces, along with their suitable feedback mechanisms, under a more complicated task. The hypothesis tested is that under a more complicated surgical task like threading, the touchless interfaces will achieve a comparable performance with touch-based ones, with respect to task efficiency (task completion time), error (needle drops), usability (number of pedal presses), and intuitiveness (learning rate). The importance of touch is influenced by the task complexity, as was found that when the level of task dexterity increases, it becomes much more difficult to finish the task with normal or top performance when in lack of somesthetic feedback (Robles-De-La-Torre, 2006, p. 28). Therefore, under this experiment, the touchless interfaces were expected to achieve a performance as good as (i.e., not outperformed by) its touch-based counterparts.

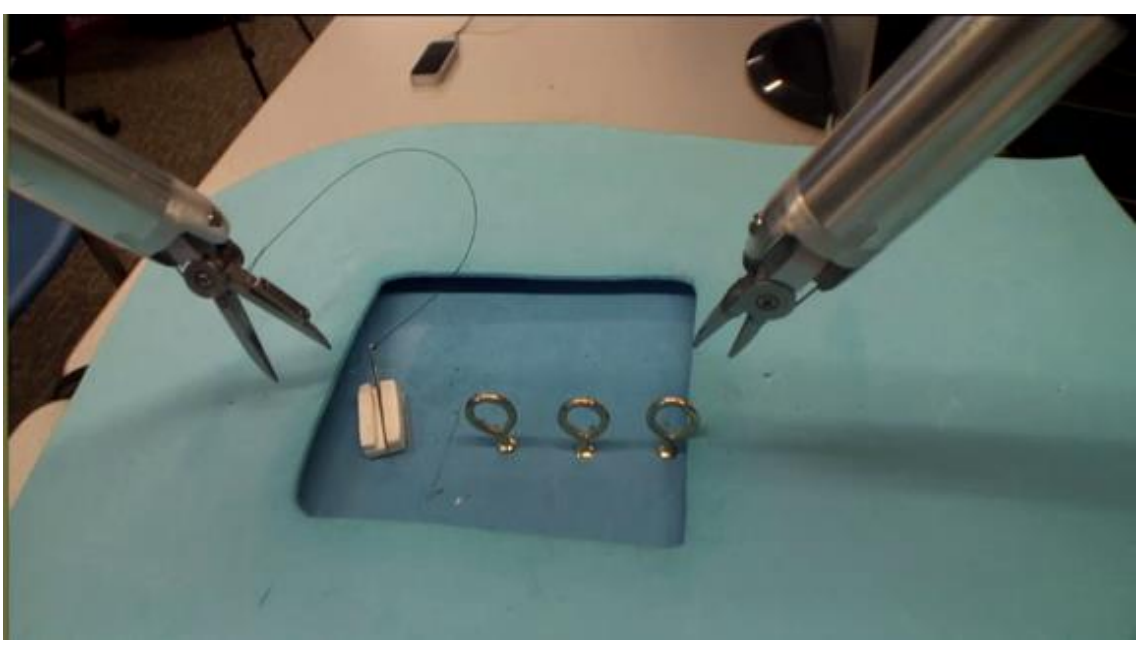

Figure 16. Threading task set-up.

\subsection{Method}

This experiment consisted of 8 engineering students, 3 males and 5 females. The selected methodology follows a within-subject experimental setup, where the operators use both interfaces (Leap Motion and Omega) to conduct the same task. The order of the interface assigned to each subject was mixed to compensate for the learning of the task (i.e., four participants used Omega first, and then Leap Motion, while the other four completed the task in the reverse order. For each interface, the task was repeated 10 times to collect enough data to find the learning curve and other metrics. The task selected in this experiment is a threading task. It consisted of having the robot thread a needle through three orifices (rings), apart from each other by $1 \mathrm{~cm}$ and positioned in a straight line. The objective was to pass the needle through each ring, while one manipulator pushed the needle from one end and the other manipulator picked it from the other end of the ring, and then pass the needle back to the first manipulator. Challenges associated with this task involved keeping the needle in straight position and controlling the yaw, pitch, and roll angles of the tooltip in way that the sewing action was accomplished without collisions with the rings or dropping the needle in the process.

\subsection{Results}

Task completion time, number of needle drops, and number of times that the foot pedal was pressed (indicates resets of the coordinate frame) were recorded during the task by the researcher. 
Leap Motion ( $M=119.2$; CI [27.5]; unit used: seconds) showed a longer task completion time than Omega ( $M=72.5$; CI [15.5]; unit used: seconds) with statistical significance $(p<0.05)$. Omega also exhibited fewer needle drops and number of pedal presses required to complete the task as compared against Leap Motion, but no significance was found. The task completion time over trials was used to obtain a learning curve for each interface. From the learning curves, learning rates were computed. Users working with Leap Motion and Omega had a learning rate (LR) of $82.82 \%$ and $83.46 \%$, respectively (no distinguishable difference). Detailed results are shown in Table 2.

Table 2. Average performance for the threading task. Only task completion time was found to be significantly different between the two groups $(\mathrm{p}<0.05)$.

\begin{tabular}{|c|c|c|c|c|}
\hline Interface & $\begin{array}{c}\text { Completion Time } \pm \\
\text { CI }(\mathbf{s})\end{array}$ & $\begin{array}{c}\text { Needle Drops } \pm \\
\text { CI }\end{array}$ & $\begin{array}{c}\text { Working Space } \\
\text { Switching } \pm \text { CI }\end{array}$ & $\begin{array}{c}\text { Learning } \\
\text { Rate }\end{array}$ \\
\hline Leap Motion & $119.2 \pm 27.5$ & $0.98 \pm 0.55$ & $11.29 \pm 3.1$ & $82.82 \%$ \\
\hline Omega & $72.5 \pm 15.5$ & $0.16 \pm 0.26$ & $4.51 \pm 1.65$ & $83.46 \%$ \\
\hline
\end{tabular}

The results found indicate that the task completion time was shorter for the Omega than for the touchless interface, with statistical significance (matched pair t-tests, two sided, $p<0.05$ ). In terms of the learning rate, it was found that LR was lower for Leap Motion than for Omega, which indicates a faster learning rate. However, the difference was not large enough to draw conclusions of dominance between them.

Regarding the number of needle drops, it was found that Omega led to a fewer number of drops than did Leap Motion. No significant difference was found.

For the number of times that the user shifted the coordinate frame of the robot, using Omega resulted in fewer times as compared to using Leap Motion. Here also, there was no statistical difference.

\section{General Discussion}

The first experiment involved a guided surgical incision while maintaining a stable depth. The resulting trajectory was compared against the reference trajectory to find the average deviation error, and the fluctuation of depth trajectory was calculated to measure the stability. A Welch's ANOVA test revealed that the deviation error using keyboard, Leap Motion, and Omega were much smaller than those using Hydra and Kinect, with statistical significance $(p<0.001)$. The comparable deviation between Leap Motion and Omega may imply that touchless interfaces can provide an equivalently accurate control, similar to the touch-based counterparts. For the analysis of control stability, the depth fluctuation of using Kinect and keyboard interfaces was much smaller than using the other interfaces $(p<0.001)$. This can be attributed to the fact that both interfaces rely on similar control schemes, in which the operator must explicitly evoke motionassociated commands gradually, one after the other, thus resulting in an operational constraint.

In the other cases, the absolute position of an operator's hands is mapped to the robot's tooltip position in the robot space automatically. Responses are faster; however, users may move their hands up or down inadvertently during a horizontal incision in the $x-y$ plane, resulting in undesirable outcomes (deeper or lighter incisions than required).

In the structured variability analysis, the higher the variance per DOF, the more control freedom the operator has through the teleoperation task. Leap Motion showed the largest RMS value (44.5), which indicates the user's larger variability of movements, implying a higher degree of naturalness in arm movements. The last interface in ranking order was the keyboard (7.6). Using the keyboard requires constrained movements (the location of the keys are fixed), and therefore, the structured variability is low (constrained arms motion). 
The second experiment involved a peg transfer task, common in laparoscopic surgery skill assessment. Experiment results revealed that touch-based interfaces led to shorter completion times than their touchless counterparts. Similarly, the number of drops observed when using Leap Motion and Kinect was higher than seen for the other three interfaces $(p<0.001)$. The "mistakes" associated with the use of Leap Motion are explained by the fact that the working space required by the Leap Motion is very limited and easy to violate. Losing track of the hands brings the tooltip to the default state "open," causing more object droppings. The fact that all the touchless interfaces were part of the Pareto set shows that these interfaces are often desired over the more traditional ones under certain performance criteria. This finding is encouraging, and more research should be devoted to understanding the reasons for this choice.

In the third experiment, we focused on completion time, learning rate, and the number of times that the user reset the reference frame of coordinates. Completion time and learning rate were discussed earlier, and they were used in this task in a similar manner. We also incorporated the last metric, number of times that the user resets the reference coordinate frame. This reflected how frequently the users reached the limit of the physical operation envelope. For the Omega, this operational envelope is very limited (when the Omega's stylus is fully extended the total diameter is about $20 \%$ larger than when the stylus holders are collapsed). As opposed to that, the physical boundary of the hand movements used with Leap Motion is limited only by the length of the human arms. In this last condition, the limit is the reflection space of the sensor (which is much smaller than the user's physical space). Due to these differences in the operational space, we expected to see that the user resets the coordinate frame of the robot many more times when using the Omega interface compared to the touchless counterpart.

The initial hypothesis made in this paper was that touchless interfaces would lead to better performance than touch-based ones, since they can provide a higher level of naturalness and freedom of motion, enabling surgeons to perform gestures similar to real gestures used in surgery. Intuitively, these are clear benefits to robotic telesurgery. After the three experiments were conducted and analyzed, we were not able to prove the validity of the initial hypothesis. Generally speaking, what we found was that touchless interfaces can match the performance of the touchbased ones in many of the metrics but could not outperform them. There are mainly two reasons for this observation. On one hand, current touchless technologies are not mature enough (e.g., lack robustness, leads to fatigue) to compete with the equivalent touch-based control interfaces. On the other hand, users are willing to trade naturalness and freedom of motion for other advantages, such as tactile sensation perceived during surgery.

The first experiments indicated that both touch-based and touchless interfaces are comparably useful. Each was better than the other on different metrics. While the values in each metric were not similar, there was no absolute solution that dominated the others (i.e., the Pareto solutions). The conclusion in this case is that either interface can be selected based on the particular preferences of the decision-maker, as often occurs in multi-objective problems.

However, in the last experiment, it was found that the Omega interface was superior in all the performance metrics compared to its touchless counterpart. This does not mean that touch-based interaction is better in general, but rather, that the current touchless technology available in the market cannot meet the performance level obtained by certain touch-based interfaces (e.g., Omega), especially in the context of complex dexterous tasks. For example, most of the needle drops in the Leap Motion scheme happen due to loss of hand tracking, hands leaving sensor range, or finger recognition mismatch.

The second reason for which we see a significant deterioration in performance in the touchless interface is the complexity of the task. All three tasks selected in this paper are surgically related tasks used for training medical students, as found in the literature (Dulan et al., 
2012a; Nielsen et al., 2004). They require different levels of dexterity and expertise from the surgeons (Dulan et al., 2012a, 2012b; Dunkin \& Wilcox, 2014), with complexity level increasing from incision to peg transfer, and lastly, threading. Fundamentals in Laparoscopic Surgery (FLS) (Dunkin \& Wilcox, 2014) also has different completion levels, with task complexities associated to test various surgical duties. Therefore, the experimental tasks in this paper were selected and performed in the order and according to the ones used for FLS.

Specifically, the threading task is much more difficult than the peg and pole transfer and incision. It requires a level of dexterity significantly higher than in the previous experiments. The complexity of the task thus leads to a more accentuated difference among the interfaces. The Omega interface allows simpler transitions between the pitch-yaw-roll motions than those found through the Leap Motion control scheme.

\section{Conclusions}

In this work, we conducted a comparative and exploratory study among touch-based and touchless interfaces selected to teleoperate a highly dexterous surgical robot. Currently, widely adopted telerobotic systems applied for surgery rely mostly on touch-based interfaces in which the surgeon's hands serve as end-effectors to give navigational commands; this approach does not include the expressiveness and naturalness found in direct-hand gesture based surgery. These gestures reflect skillful action gestures and also convey intent, emotions, and content between members of a surgical team present in an operating room. For this reason, studying the feasibility to incorporate new technologies that allow free hand gestures opens a new venue for surgical interfaces. We implemented and tested a system using three experimental designs, based on standardized surgically related tasks for medical training in laparoscopic surgeries.

Several clinically relevant performance metrics were measured to evaluate the user's task performance, learning rates, control stability, and interaction naturalness. Experimental results revealed that when task complexity varies from simple to medium, either interface type can be used since their performance is comparable (no interface dominates the other in every performance metric). In such a case, the decision-maker (e.g., surgeon) has to make the final decision. Nevertheless, for the most complex task, it seems that the touch-based interface exhibits better performance than its counterpart. This result does not mean that touchless interfaces are not suitable for this type of task; but that touchless interfaces are still under ongoing development. Yet, an important aspect of this research is to show the feasibility of gaming technologies as means of control and teleoperation in a variety of challenging tasks, and to test the concept of sensorial substitution in touchless teleoperation. This research also surveyed state of the art of touchless technologies and their applicability to the surgical setting. These interfaces, and also the more traditional ones, were discussed, and advantages and limitations of each were presented in the context of the surgical setting. This work can serve decision makers, technologists, and clinicians as a fundamental baseline for further development and adoption of natural, direct, and expressive modalities of interaction between humans and robots in the clinical setting.

\section{Acknowledgements}

This publication was made possible by NPRP award 6-449-2-181 from the Qatar National Research Fund (a member of the Qatar Foundation). The statements made herein are solely the responsibility of the authors. We would like to thank our fellow Intelligent Systems and Assistive Technologies lab members at Purdue for their helpful suggestions, tips, and guidance. In addition, we would also like to acknowledge the people at SRI International for their support throughout this entire project. 


\section{References}

Bach-y-Rita, P., \& W. Kercel, S. (2003). Sensory substitution and the human-machine interface. Trends in Cognitive Sciences, 7(12), 541-546.

Beasley, R. A. (2012). Medical robots: Current systems and research directions. Journal of Robotics, 2012, e401613.

Carriere, K. C., \& Reinsel, G. C. (1992). Investigation of dual-balanced crossover designs for two treatments. Biometrics, 48(4), 1157-1164.

Carrino, F., Ridi, A., Mugellini, E., Khaled, O. A., \& Ingold, R. (2012). Gesture segmentation and recognition with an EMG-based intimate approach-An accuracy and usability study. In Proceedings of the 2012 Sixth International Conference on Complex, Intelligent and Software Intensive Systems (CISIS) (pp. 544-551). Palermo, Italy.

Carswell, C. M., Clarke, D., \& Seales, W. B. (2005). Assessing mental workload during laparoscopic surgery. Surgical Innovation, 12(1), 80-90.

da Vinci Surgery-Minimally Invasive Robotic Surgery With the da Vinci Surgical System. (2015). Retrieved on April 19, 2015 from http://www.davincisurgery.com

DeSouza, G. N., Jiang, H., Wachs, J. P., \& Duerstock, B. S. (2014). Integrated vision-based system for efficient, semi-automated control of a robotic manipulator. International Journal of Intelligent Computing and Cybernetics, 7(3), 253-266.

Dulan, G., Rege, R. V., Hogg, D. C., Gilberg-Fisher, K. M., Arain, N. A., Tesfay, S. T., \& Scott, D. J. (2012a). Developing a comprehensive, proficiency-based training program for robotic surgery. Surgery, 152(3), 477-488.

Dulan, G., Rege, R. V., Hogg, D. C., Gilberg-Fisher, K. M., Arain, N. A., Tesfay, S. T., \& Scott, D. J. (2012b). Proficiency-based training for robotic surgery: Construct validity, workload, and expert levels for nine inanimate exercises. Surgical Endoscopy, 26(6), 1516-1521.

Dunkin, B., \& Wilcox, V. (2014). Developing a curriculum for residents and fellows. In K. C. Kim (Ed.), Robotics in general surgery (pp. 385-413). New York: Springer.

Force Dimension (2015). Products-Omega.7. Retrieved on August 26, 2016 from http://www.forcedimension.com/products/omega-7/overview

Frankel, A. S., Leonard, M. W., \& Denham, C. R. (2006). Fair and just culture, team behavior, and leadership engagement: The tools to achieve high reliability. Health Services Research, 41(4p2), 1690-1709.

Greer, A. D., Newhook, P. M., \& Sutherland, G. R. (2008). Human-machine interface for robotic surgery and stereotaxy. IEEE/ASME Transactions on Mechatronics, 13(3), $355-361$.

Hartmann, B., Benson, M., Junger, A., Quinzio, L., Röhrig, R., Fengler, B., Hempelmann, G. (2004). Computer keyboard and mouse as a reservoir of pathogens in an intensive care unit. Journal of Clinical Monitoring and Computing, 18(1), 7-12.

Hoshi, T., Takahashi, M., Nakatsuma, K., \& Shinoda, H. (2009). Touchable holography. In Proceedings of the ACM SIGGRAPH Conference on Emerging Technologies (pp. 23:123:1). New York, NY: ACM.

Huang, J.-D. (2011). Kinerehab: A Kinect-based system for physical rehabilitation: A pilot study for young adults with motor disabilities. In Proceedings of the 13th International 
ACM SIGACCESS Conference on Computers and Accessibility (pp. 319-320). New York, NY: ACM.

Hu, J., Edsinger, A., Lim, Y.-J., Donaldson, N., Solano, M., Solochek, A., \& Marchessault, R. (2011). An advanced medical robotic system augmenting healthcare capabilitiesRobotic nursing assistant. In Proceedings of the IEEE International Conference on Robotics and Automation (ICRA) (pp. 6264-6269). Shanghai, China.

Jacob, M., Cange, C., Packer, R., \& Wachs, J. P. (2012). Intention, context and gesture recognition for sterile MRI navigation in the operating room. In L. Alvarez, M. Mejail, L. Gomez, \& J. Jacobo (Eds.), Progress in pattern recognition, image analysis, computer vision, and applications (pp. 220-227). Berlin Heidelberg, Germany: Springer.

Jacob, M. G., Li, Y.-T., \& Wachs, J. P. (2011). A gesture driven robotic scrub nurse. In Proceedings of the IEEE International Conference on Systems, Man, and Cybernetics (SMC) (pp. 2039-2044). Anchorage, AK.

Jacob, M. G., \& Wachs, J. P. (2014). Context-based hand gesture recognition for the operating room. Pattern Recognition Letters, 36, 196-203.

Jacob, M. G., Wachs, J. P., \& Packer, R. A. (2013). Hand-gesture-based sterile interface for the operating room using contextual cues for the navigation of radiological images. Journal of the American Medical Informatics Association, 20(e1), e183-e186.

Jaimes, A., \& Sebe, N. (2005). Multimodal human computer interaction: A survey. In N. Sebe, M. Lew, \& T. S. Huang (Eds.), Computer vision in human-computer interaction (pp. 115). Berlin Heidelberg, Germany: Springer.

Jakus, G., Guna, J., Tomažič, S., \& Sodnik, J. (2014). Evaluation of leap motion controller with a high precision optical tracking system. In M. Kurosu (Ed.), Human-computer interaction. Advanced interaction modalities and techniques (pp. 254-263). Switzerland: Springer International Publishing.

Kim, Y., Kim, P. C. W., Selle, R., Shademan, A., \& Krieger, A. (2014). Experimental evaluation of contact-less hand tracking systems for tele-operation of surgical tasks. In Proceedings of the IEEE International Conference on Robotics and Automation (ICRA) (pp. 3502-3509). Hong Kong, China.

Kim, Y., Leonard, S., Shademan, A., Krieger, A., \& Kim, P. C. W. (2014). Kinect technology for hand tracking control of surgical robots: Technical and surgical skill comparison to current robotic masters. Surgical Endoscopy, 28(6), 1993-2000.

Kinect (2015). Meet Kinect for Windows. Retrieved on August 26, 2016 from https://developer.microsoft.com/en-us/windows/kinect

Kitagawa, M., Dokko, D., Okamura, A. M., \& Yuh, D. D. (2005). Effect of sensory substitution on suture-manipulation forces for robotic surgical systems. The Journal of Thoracic and Cardiovascular Surgery, 129(1), 151-158.

Lakoff, G., \& Johnson, M. (2003). Metaphors we live by (2nd Ed.). Chicago: University Of Chicago Press.

Leap Motion (2015). Mac \& PC motion controller for games, design, \& more. Retrieved on April 28, 2015 from https://www.leapmotion.com 
Lingard, L., Reznick, R., Espin, S., Regehr, G., \& DeVito, I. (2002). Team communications in the operating room: Talk patterns, sites of tension, and implications for novices. Academic Medicine: Journal of the Association of American Medical Colleges, 77(3), 232-237.

Mann, R. W. (1974). Technology and human rehabilitation: Prostheses for sensory rehabilitation and/or sensory substitution. In J. Dickson (Ed.), Advances in Biomedical Engineering (pp. 209-353). Cambridge, MA: Academic Press.

Mitra, S., \& Acharya, T. (2007). Gesture recognition: A survey. IEEE Transactions on Systems, Man, and Cybernetics, Part C: Applications and Reviews, 37(3), 311-324.

Monnich, H., Nicolai, P., Beyl, T., Raczkowsky, J., \& Worn, H. (2011). A supervision system for the intuitive usage of a telemanipulated surgical robotic setup. In Proceedings of the IEEE International Conference on Robotics and Biomimetics (ROBIO) (pp. 449454). Phuket Island, Thailand.

Moore, A., Butt, D., Ellis-Clarke, J., \& Cartmill, J. (2010). Linguistic analysis of verbal and non-verbal communication in the operating room. ANZ Journal of Surgery, 80(12), 925-929.

Myo (2015). Myo gesture control armband. Retrieved on August 26, 2016 from https://www.myo.com

Nehaniv, C. L., Dautenhahn, K., Kubacki, J., Haegele, M., Parlitz, C., \& Alami, R. (2005). A methodological approach relating the classification of gesture to identification of human intent in the context of human-robot interaction. In Proceedings of the IEEE International Workshop on Robot and Human Interactive Communication (ROMAN). (pp. 371-377). Nashville, TN.

Nielsen, M., Störring, M., Moeslund, T. B., \& Granum, E. (2004). A Procedure for developing intuitive and ergonomic gesture interfaces for HCI. In A. Camurri \& G. Volpe (Eds.), Gesture-based communication in human-computer interaction (pp. 409-420). Berlin Heidelberg: Springer.

Nisky, I., Hsieh, M. H., \& Okamura, A. M. (2013). A framework for analysis of surgeon arm posture variability in robot-assisted surgery. In Proceedings of the IEEE International Conference on Robotics and Automation (ICRA) (pp. 245-251). Karlsruhe, Germany.

O’Hara, K., Harper, R., Mentis, H., Sellen, A., \& Taylor, A. (2013). On the Naturalness of Touchless: Putting the "Interaction" Back into NUI. ACM Transactions on ComputerHuman Interaction, 20(1), 5:1-5:25.

Oikonomidis, I., Kyriazis, N., \& Argyros, A. A. (2011). Efficient model-based 3D tracking of hand articulations using Kinect. In British Machine Vision Conference (BMVC) (pp. 111). Dundee, Scotland.

Oviatt, S. (2006). Human-centered design meets cognitive load theory: Designing interfaces that help people think. In Proceedings of the 14th Annual ACM International Conference on Multimedia (pp. 871-880). New York, NY: ACM.

Pavlovic, V. I., Sharma, R., \& Huang, T. S. (1997). Visual interpretation of hand gestures for human-computer interaction: A review. IEEE Transactions on Pattern Analysis and Machine Intelligence, 19(7), 677-695.

Prentice, R. (2007). Drilling surgeons the social lessons of embodied surgical learning. Science, Technology \& Human values, 32(5), 534-553. 
Rautaray, S. S., \& Agrawal, A. (2012). Vision based hand gesture recognition for human computer interaction: A survey. Artificial Intelligence Review, 1-54.

Razor Inc. (2015). Enter the Hydra Razer ${ }^{\mathrm{TM}}$ for Gamers. By Gamers. ${ }^{\mathrm{TM}}$. Retrieved on April 28, 2015 from http://www.razerzone.com/minisite/hydra

Robles-De-La-Torre, G. (2006). The importance of the sense of touch in virtual and real environments. IEEE Multimedia, 13(3), 24-30.

Rosen, J., Hannaford, B., \& Satava, R. M. (2011). Surgical robotics: Systems applications and visions. New York: Springer Science \& Business Media, 2011.

Rovira, K., Gapenne, O., \& Ammar, A. A. (2010). Learning to recognize shapes with a sensory substitution system: A longitudinal study with 4 non-sighted adolescents. In Proceedings of the IEEE 9th International Conference on Development and Learning (ICDL) (pp. 1-6). Ann Arbor, MI.

Schultz, M., Gill, J., Zubairi, S., Huber, R., \& Gordin, F. (2003). Bacterial contamination of computer keyboards in a teaching hospital. Infection Control and Hospital Epidemiology, 24(4), 302-303.

Sengül, A., van Elk, M., Rognini, G., Aspell, J. E., Bleuler, H., \& Blanke, O. (2012). Extending the body to virtual tools using a robotic surgical interface: Evidence from the crossmodal congruency task. PLoS One, 7(12), e49473.

Sodhi, R., Poupyrev, I., Glisson, M., \& Israr, A. (2013). AIREAL: Interactive tactile experiences in free air. ACM Transactions on Graphics (TOG)—SIGGRAPH 2013 Conference Proceedings, 32(4), 134:1-134:10.

Talamini, M., Campbell, K., \& Stanfield, C. (2002). Robotic gastrointestinal surgery: Early experience and system description. Journal of Laparoendoscopic \& Advanced Surgical Techniques Part A., 12(4), 225-232.

Visell, Y. (2009). Tactile sensory substitution: Models for enaction in HCI. Interacting With Computers, 21(1-2), 38-53.

von Hardenberg, C., \& Bérard, F. (2001). Bare-hand human-computer interaction. In Proceedings of the 2001 Workshop on Perceptive User Interfaces (pp. 1-8). New York, NY: ACM.

Wachs, J. P., Kölsch, M., Stern, H., \& Edan, Y. (2011). Vision-based hand-gesture applications. Communications of the ACM, 54(2), 60-71.

Wachs, J. P., Stern, H. I., Edan, Y., Gillam, M., Handler, J., Feied, C., \& Smith, M. (2008). A gesture-based tool for sterile browsing of radiology images. Journal of the American Medical Informatics Association, 15(3), 321-323.

Wagner, C. R., Stylopoulos, N., Jackson, P. G., \& Howe, R. D. (2007). The benefit of force feedback in surgery: Examination of blunt dissection. Presence: Teleoperators and Virtual Environments, 16(3), 252-262.

Wallenstein, S., \& Fisher, A. C. (1977). The analysis of the two-period repeated measurements crossover design with application to clinical trials. Biometrics, 33(1), 261-269.

Webster, J. L., \& Cao, C. G. L. (2006). Lowering communication barriers in operating room technology. Human Factors: The Journal of the Human Factors and Ergonomics Society, 48(4), 747-758. 
Weichert, F., Bachmann, D., Rudak, B., \& Fisseler, D. (2013). Analysis of the accuracy and robustness of the Leap Motion controller. Sensors, 13(5), 6380-6393.

Whittaker, S. (2003). Theories and methods in mediated communication. In The handbook of discourse processes (pp. 243-286). Mahwah, NJ: Erlbaum.

Zhou, T., Cabrera, M. E., \& Wachs, J. (2014). Touchless telerobotic surgery-A comparative study. In IEEE IROS in 3rd Workshop on Telerobotics for Real-Life Applications, Opportunities, Challenges and New Developments. Chicago, IL.

Zhou, T., Cabrera, M. E., \& Wachs, J. P. (2015). Touchless telerobotic surgery-Is it possible at all? In Proceedings of the 29th AAAI Conference on Artificial Intelligence. Austin, TX.

Zubrycki, I., \& Granosik, G. (2014). Using integrated vision systems: Three gears and Leap Motion, to control a 3-finger dexterous gripper. In R. Szewczyk, C. Zieliński, \& M. Kaliczyńska (Eds.), Recent advances in automation, robotics and measuring techniques (pp. 553-564). Switzerland: Springer International Publishing.

T. Zhou, Purdue University, West Lafayette, IN, USA. Email: zhou338@purdue.edu; M. E. Cabrera, Purdue University, West Lafayette, IN, USA. Email: cabrerm@purdue.edu; T. Low, SRI International, Menlo Park, CA, USA. Email: thomas.low@sri.com; C. Sundaram, Indiana University, Indianapolis, IN, USA. Email: sundaram@iupui.edu; J. P. Wachs, Purdue University, West Lafayette, IN, USA. Email: jpwachs@ purdue.edu. 